TITLE:

\title{
Interconnected Turing patterns in three dimensions
}

\author{
$\operatorname{AUTHOR}(\mathrm{S})$ :
}

Shoji, H; Yamada, K; Ohta, T

\section{CITATION:}

Shoji, H ...[et al]. Interconnected Turing patterns in three dimensions. Physical Review E 2005, 72(6): 065202.

ISSUE DATE:

2005-12

URL:

http://hdl.handle.net/2433/49963

RIGHT:

Copyright 2005 American Physical Society 
PHYSICAL REVIEW E 72, 065202(R) (2005)

\title{
Interconnected Turing patterns in three dimensions
}

\author{
Hiroto Shoji, ${ }^{1}$ Kohtaro Yamada, ${ }^{1,2}$ and Takao Ohta ${ }^{1}$ \\ ${ }^{1}$ Yukawa Institute for Theoretical Physics, Kyoto University, Kyoto 606-8502, Japan \\ ${ }^{2}$ Department of Mathematical and Life Sciences, Graduate School of Science, Hiroshima University, Higashi-Hiroshima 739-8526, Japan
}

(Received 20 September 2005; published 20 December 2005)

\begin{abstract}
We study numerically the Turing pattern in three dimensions in a FitzHugh-Nagumo-type reaction-diffusion system. We have found that interconnected periodic domain structures such as a gyroid, $F d d d$, and perforated lamellar structures appear in three dimensions, which never exist in lower dimensions. The stability analysis of these structures is also performed by means of a mode expansion.
\end{abstract}

DOI: 10.1103/PhysRevE.72.065202

PACS number(s): 89.75.Kd, 47.54.+r, 05.70.Ln

More than 50 years ago, Turing showed that a coupled reaction-diffusion equation with two components admits spatially periodic solutions if certain conditions are fulfilled [1]. The mechanism is now called a diffusion-driven instability or Turing instability. For five decades, Turing patterns have been studied numerically and mathematically as an example of dissipative structures far from equilibrium [2]. Pattern formation in various biological systems has also been accounted for by the Turing mechanism [3]. Experimental evidence of a Turing structure was observed in an open chemical reactor $[4,5]$. However, almost all of the previous investigations are restricted to one or two dimensions, where only stripe, hexagonal, and labyrinthine patterns are concerned.

In this Rapid Communication, we study Turing structures in three dimensions. It is emphasized that there are essentially new structures in three dimensions apart from the corresponding two-dimensional structures. An example is the interconnected periodic domains whose surface consists of a minimal surface such that the average curvature is zero everywhere on the surface. This kind of pattern can never exist in two dimensions.

De Wit et al. [6] were studied Turing structures in three dimensions by computer simulations. Later, De Wit et al. [7] and Leppanen et al. [8] found numerically spherical domains in a body-centered-cubic (bcc) lattice, hexagonally packed cylinders, lamellar, and distorted lamellar structures. By comparing dissipative structures with those arising in soft matter physics, the possibilities of formation of periodic minimal surfaces not only at the grain boundaries but also in a bulk have also been discussed [7]. A group-theoretical analysis of three-dimensional Turing patterns has been carried out by Callahan and Knobloch [9].

The model which we shall explore is the following FitzHugh Nagumo-type reaction-diffusion equation [10]:

$$
\begin{gathered}
\frac{\partial u}{\partial t}=D_{u} \nabla^{2} u+u-u^{3}-v, \\
\frac{\partial v}{\partial t}=D_{v} \nabla^{2} v+\gamma(u-\alpha v-\beta),
\end{gathered}
$$

where the constants $\alpha, \beta, \gamma, D_{u}$, and $D_{v}$ are all positive. This set of equations has been studied as a model equation of impulse propagation along a nerve axion [11] and of the
Belousov-Zhabotinsky chemical reaction [12]. Equations (1) and (2) have a stable solution periodic in space if the parameters are chosen such that the system without the diffusion terms has only one equilibrium solution, $(\bar{u}, \bar{v})$ which is given by $\bar{u}-\bar{u}^{3}-\bar{v}=0$ and $\bar{u}-\alpha \bar{v}-\beta=0$. The linear stability analysis of the uniform solution is readily carried out. By setting $(u-\bar{u}, v-\bar{v}) \sim \exp (i k x+\lambda t)$, we obtain the eigenvalue $\lambda$ as a function of the wave number $k$. It is found that if $D_{u} \ll D_{v}$ there is a bifurcation threshold such that the eigenvalue becomes positive for a finite value of $k=k_{c}$.

We have carried out numerical simulations for the coupled set of Eqs. (1) and (2) in three dimensions. The space is divided into $L^{3}$ cubic cells of size $L=32$ and the periodic boundary conditions are imposed at the system boundaries. The simple Euler algorithm is used with time step $\delta t=0.0070$. In order to remove the anisotropy in discretizing the Laplacian, we have employed a 27-point difference scheme [13].

One of the most crucial technical points is to determine the spatial period of periodic structures. Although the critical wave number $k_{c}>0$ at the bifurcation point can be obtained by the linear stability analysis, the true period at postthreshold is impossible to accurately evaluate analytically. We have repeated simulations by changing the cell size $\delta x$ and found that $\delta x=5.3 \times 10^{-3}$ (the linear dimension of the system is equal to $32 \times \delta x=0.17$ ) is mostly commensurate with the periodic structures. We have verified that the results shown below are unaltered for $L=50$ with $\delta x=3.4 \times 10^{-3}$ and $L$ $=64$ with $\delta x=2.7 \times 10^{-3}$. If the system size is large enough compared to the spatial period, many grains appear in numerical simulations. Those are almost frozen so that it is

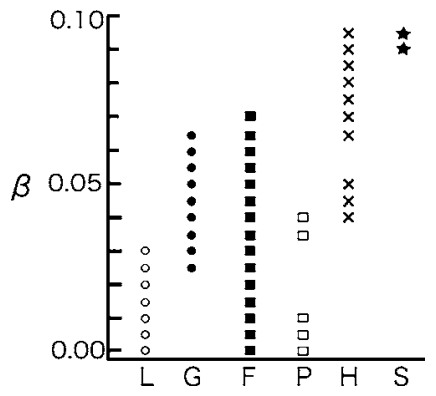

FIG. 1. Five stationary solutions. The meanings of $L, G, F, P$, $H$, and $S$ are given in the text. 

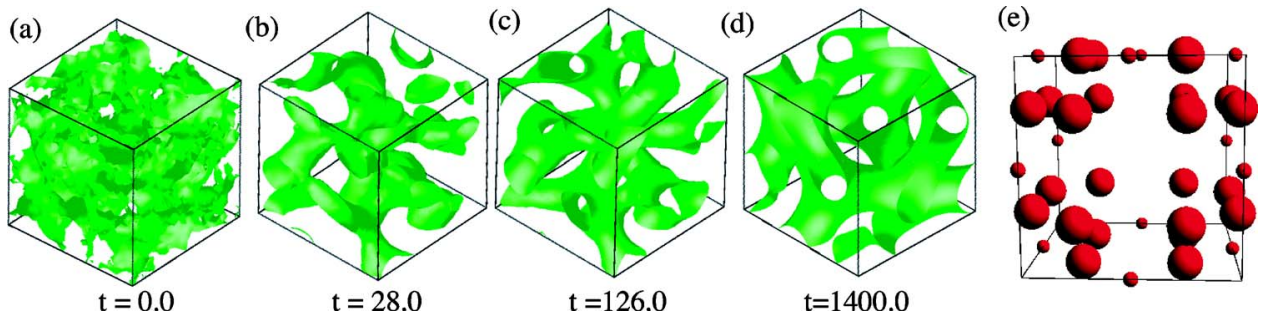

FIG. 2. (Color online) (a)-(d) Structural evolution of domains for $\beta=0.04$ and (e) the Bragg positions of the asymptotic structure. For the sake of clarity, the domains in (a) represent the isosurface of $u=0.08$ whereas those in (b)-(d) represent the isosurface of $u=0.05$. (e) The radius of the spheres is proportional to the relative peak intensity. In this figure, Fig. 3(b), and Fig. 4(b), the Bragg point at the origin is omitted.

difficult to obtain the final most stable structure. In order to avoid this, the system size is chosen to be the same as the unit cell of the structure.

Numerical simulations are performed for $D_{u}=5.0 \times 10^{-5}$, $D_{v}=5.0 \times 10^{-3}, \alpha=0.5, \gamma=26$, and changing the parameter $\beta$ [14]. We start with the unstable uniform solution $(\bar{u}, \bar{v})$ with small random perturbation superimposed. The random force is added to Eqs. (1) and (2) to remove trapping at the metastable states. The asymptotic stationary solutions obtained are summarized in Fig. 1. This phase diagram was obtained by changing the random initial conditions for a given value of $\beta$. Note that two or three different structures coexist. The abbreviations $L, H$, and $S$ mean lamellar, hexagonal, and bcc structures, respectively. The remaining three structures $G, F$, and $P$ are analyzed below.

The formation of $G$ is displayed in Fig. 2. We have carried out a Fourier transformation of the asymptotic values of $u$ and $v$, and obtained the Bragg peaks which are listed in Table I together with the peak intensities. The peak position is defined by $\vec{k} L / 2 \pi$ with $\vec{k}$ the wave number vector. The third peaks are not shown in Table I because those intensities $I_{k}$ $=\left|u_{k}\right|^{2} \cong 21$ are quite small. From these results we identify $G$ with a gyroid structure. It is well known that a gyroid structure can be approximated by the following level set equation

$$
\begin{aligned}
0= & 8(1-\eta)[\sin 2 x \sin z \cos y+\sin 2 y \sin x \cos z \\
& +2 \sin 2 z \sin y \cos x]-4 \eta[\cos 2 x \cos 2 y+\cos 2 y \cos 2 z \\
& +\cos 2 z \cos 2 x]-\xi,
\end{aligned}
$$

TABLE I. The Bragg peak positions and their intensities (arbitrary unit) of a gyroid structure. In this table and Table II, the Bragg positions that are symmetric with respect to the origin are omitted.

\begin{tabular}{cccc}
\hline \hline Peak Position & $I_{k}$ & Peak Position & $I_{k}$ \\
\hline$(2,1,1)$ & 1599.49 & $(1,-1,-2)$ & 1599.78 \\
$(2,1,-1)$ & 1599.85 & $(1,-2,1)$ & 1599.73 \\
$(2,-1,1)$ & 1599.83 & $(1,-2,-1)$ & 1599.76 \\
$(2,-1,-1)$ & 1599.66 & $(2,2,0)$ & 589.813 \\
$(1,2,1)$ & 1599.79 & $(2,0,2)$ & 589.858 \\
$(1,2,-1)$ & 1599.50 & $(2,0,-2)$ & 589.935 \\
$(1,1,2)$ & 1599.82 & $(2,-2,0)$ & 589.889 \\
$(1,1,-2)$ & 1599.72 & $(0,2,2)$ & 589.931 \\
$(1,-1,2)$ & 1599.51 & $(0,2,-2)$ & 589.779 \\
\hline \hline
\end{tabular}

where $\eta$ and $\xi$ are the parameters [15]. The Bragg positions evaluated numerically are completely consistent with the reciprocal lattice vectors obtained from Eq. (3). The asymptotic domain structure of $F$ and the Bragg positions are shown in Fig. 3. The Bragg peaks are listed together with the peak intensities in Table II. We omitted the Bragg peaks for $I_{k} \leqslant 100$. From these analyses we conclude that this structure is an Fddd structure. Similarly, the stationary structure of $P$ is displayed in Fig. 4 together with the Bragg positions. The intensity of the higher order peaks decreases more slowly than those of the $F d d d$ structure. This is a layer structure having holes in each layer and is identified with the so called perforated lamellar structure.

As mentioned above, several structures can coexist for a fixed set of parameters. One of the basic questions is to determine the most stable structure. However this is highly nontrivial because Eqs. (1) and (2) are nonvariational with no Lyapunov functional. Probably the general method to see the relative stability of two different structures is to examine the motion of a planer interface separating those structures as was attempted in [8] for a bistable reaction-diffusion system. If one of the structures invades the other, one may conclude that the former is more stable than the latter. However to achieve this, one needs to provide a sufficiently large system for numerical simulations. This is beyond our computer facilities.

Here we employ two methods to examine the stability. One is to explore numerically the volume of the basin of each stable structure. To this end, we have carried out the

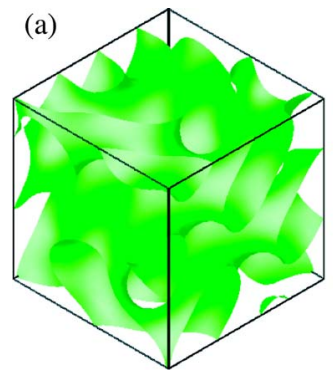

(b)
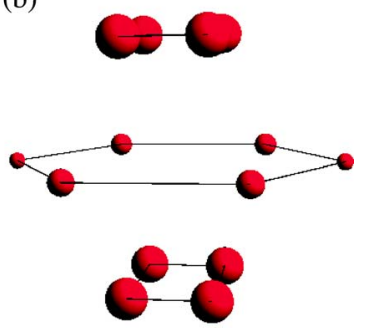

FIG. 3. (Color online) $F d d d$ structure for $\beta=0.04$ represented by the isosurface of $u=0.05$ and (b) the Bragg positions with $I_{k}$ $>800$ in Table II. The details of (b) are the same as those in Fig. 2(e). The Bragg points on the same plane are connected with the line. It is noted that the quasiproper hexagon and the two rectangles are parallel to each other. 
TABLE II. The Bragg peak positions and their intensities (arbitrary unit) of an Fddd structure.

\begin{tabular}{cccc}
\hline \hline Peak Position & $I_{k}$ & Peak Position & $I_{k}$ \\
\hline$(1,2,1)$ & 2817.23 & $(1,1,-2)$ & 145.39 \\
$(-1,1,2)$ & 2785.42 & $(1,2,-1)$ & 142.13 \\
$(1,-2,-1)$ & 2571.37 & $(1,-2,1)$ & 137.97 \\
$(1,1,2)$ & 2559.69 & $(0,2,-2)$ & 835.60 \\
$(2,1,-1)$ & 1457.85 & $(1,0,3)$ & 170.05 \\
$(2,-1,1)$ & 1297.37 & $(1,-3,0)$ & 173.25 \\
$(2,-1,-1)$ & 192.43 & $(1,0,-3)$ & 214.31 \\
$(2,1,1)$ & 179.87 & $(1,3,0)$ & 211.79 \\
$(1,-1,2)$ & 147.37 & $(4,0,0)$ & 137.61 \\
\hline \hline
\end{tabular}

following simulations. We provide the structures of $L, G, H$, $S, F$, and $P$ for a given value of $\beta$ and start numerical simulations for Eqs. (1) and (2) with a certain amplitude of random forces up to $4 \times 10^{5}$ time steps. Then, we turn off the random forces and continue numerical simulations up to the same steps as the above and see whether the initial structure appears or not. In this way, we can obtain numerically the upper limit of the noise amplitude below which the initial structure recovers. Table III shows the results for $\beta=0.04$. It is found that $G$ is mostly robust against the perturbation. Other structures do not survive even for weaker perturbation. This implies for $\beta=0.04$ that $G$ has the widest basin of attractor in the functional space. If the amplitude of noises is larger than $0.044, G$ is broken and one of the other structures $F, H$, or $L$ appears asymptotically.

The other method is to derive approximately a Lyapunov functional for Eqs. (1) and (2) [16]. In the limit $\gamma, D_{v} \rightarrow \infty$ with $\gamma / D_{v}$ finite, one may set $\partial v / \partial t=0$ in Eq. (2), so that the variable $v$ can be eliminated and one obtains

$$
\frac{\partial u}{\partial t}=-\frac{\delta F}{\delta u}
$$

where

$$
\begin{aligned}
F\{u\}= & \int d \vec{r}\left[\frac{D_{u}}{2}(\vec{\nabla} u)^{2}-\frac{u^{2}}{2}+\frac{u^{4}}{4}\right]+\frac{\gamma}{2 D_{v}} \int d \vec{r} \int d \vec{r}^{\prime} G\left(\vec{r}, \vec{r}^{\prime}\right) \\
& \times[u(\vec{r}, t)-\beta] \times\left[u\left(\vec{r}^{\prime}, t\right)-\beta\right] .
\end{aligned}
$$

The Green function is defined through

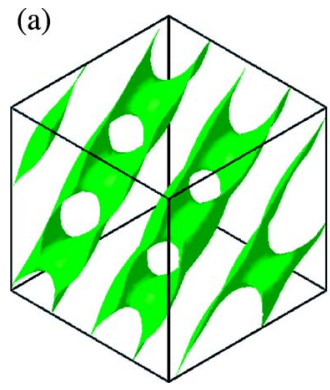

(b)

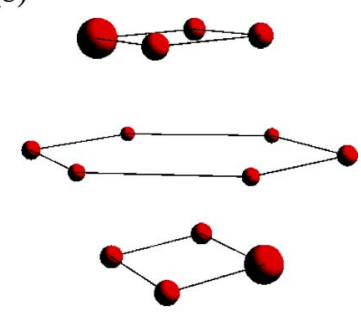

FIG. 4. (Color online) Perforated lamellar structure for $\beta$ $=0.04$ represented by the isosurface of $u=0.05$ and (b) the Bragg positions with $I_{k}>750$. The details of (b) are the same as those in Fig. 2.
TABLE III. Maximum perturbation strength beyond which the original structure does not survive at $\beta=0.04$.

\begin{tabular}{cc}
\hline \hline Structures & Strength \\
\hline Gyroid & 0.044 \\
Fddd & 0.040 \\
Hexagonal cylinder & 0.038 \\
Lamellar & 0.020 \\
bcc & 0.015 \\
$P L$ & 0.008 \\
\hline \hline$\left(-\nabla^{2}+\frac{\alpha \gamma}{D_{v}}\right) G\left(\vec{r}, \vec{r}^{\prime}\right)=\delta\left(\vec{r}-\vec{r}^{\prime}\right)$.
\end{tabular}

Since $\gamma / D_{v}$ is positive, the functional $F$ plays a role of a Lyapunov functional for the reduced equation (4).

Here we make a remark that the Lyapunov functional (5) is the same as the free energy functional derived in the study of the microphase separation of diblock copolymers [17] if $\alpha=0$ and $\beta$ is replaced by the spatial average of $u$ where $u$ has a meaning of the local concentration difference between two blocks. Since $u$ is a conserved quantity in block copolymers, the time-evolution equation is not given by Eq. (4) but by $\partial u / \partial t=\nabla^{2}(\delta F / \delta u)$.

We attempt to investigate the relative stability of Turing structures by using the Lyapunov functional (5) in the reduced system. Of course, this is an approximation for the original coupled set of Eqs. (1) and (2). However, we expect that some insights into the stability are provided by this method.

We employ the mode expansion for the variable, $u$

$$
u(\vec{r}, t)=\bar{u}+\left[\sum_{n=1}^{N} a_{n}(t) e^{i \vec{q}_{n} \cdot \vec{r}}+\text { c.c. }\right],
$$

where $a_{n}(t)$ is the real amplitudes and c.c. means a complex conjugate. The fundamental reciprocal lattice vectors are

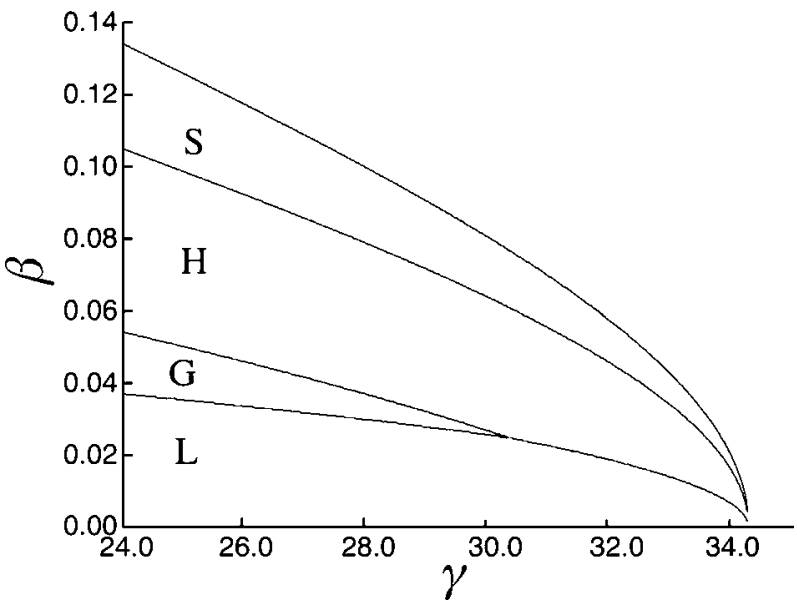

FIG. 5. Phase diagram in the $\gamma-\beta$ plane. The most stable regions for lamellar, hexagonal. gyroid and BCC are indicated by $L, H, G$ and $S$, respectively. 
listed in Table I. The number of modes is equal to $N=18$ for the gyroid structure. It is noted that lamellar, hexagonal, bcc, and $F d d d$ structures can be represented as a substructure of the gyroid in the sense that the reciprocal vectors of these structures are contained in the 18 modes. We have verified numerically that the spatial variation of the structures in Fig. 2(d) is almost sinusoidal and hence that the mode expansion trancated up to $N=18$ is justified [18]. Substituting Eq. (7) into Eq. (5) and ignoring the higher harmonics, we obtain the Lyapunov functional in terms of the amplitudes and the magnitudes of the reciprocal vectors, $F_{\text {mode }}=F\left(\left\{a_{n}\right\}, q_{n}\right)$. This explicit form is not written here because it is lengthy. Minimization of $F_{\text {mode }}$ with respect to $a_{n}$ and $q_{n}$ gives us the stationary structures and the value of the Lyapunov functional for each structure. In this way, one may determine the most stable structure for fixed parameters in the reduced system (4). The result is summarized in Fig. 5. This theoretical phase diagram is not inconsistent with numerical results in Fig. 1 for $\gamma=26$.
In summary, we have studied Turing patterns in three dimensions. Apart from lamellar and hexagonal structures which are simple generalization of two-dimensional patterns, we have obtained gyroid, $F d d d$, bcc, and perforated lamellar structures. Except for the bcc structure, the domains consist of interconnected networks which are characteristic to three dimensions. Furthermore, all of these structures have also been obtained in an entirely different problem, that is, microphase separation of diblock copolymers. In this paper, we have shown, explicitly, the relationship between the threedimensional Turing pattern far from equilibrium and microphase separation in thermal equilibrium. The results obtained here would provide deep insights into the fundamental mechanism of pattern formation in nature.

We would like to thank T. Uneyama for his advice in numerical simulations. H. S. and K. Y. are supported by a Grant-in-Aid for JSPS Fellows for Young Scientists, from the Japan Society for the Promotion of Science.
[1] A. M. Turing, Philos. Trans. R. Soc. London 237, 37 (1952).

[2] G. Nicolis and I. Prigogine, Self-Organization in Nonequilibrium Systems (Wiley, New York, 1977).

[3] J. D. Murray, Mathematical Biology, 2nd. ed. (SpringerVerlag, Berlin, 1993).

[4] V. Castets, E. Dulos, J. Boissonade, and P. De Kepper, Phys. Rev. Lett. 64, 2953 (1990)

[5] Q. Ouyang and H. L. Swinney, Nature (London) 352, 610 (1991).

[6] A. De Wit, G. Dewel, P. Borckmans, and D. Walgraef, Physica D 61, 289 (1992).

[7] A. De Wit, P. Borckmans, and G. Dewel, Proc. Natl. Acad. Sci. U.S.A. 94, 12765 (1997).

[8] T. Leppanen, M. Karttunen, K. Kaski, R. A. Barrio, and L. Zhang, Physica D 168, 35 (2002); T. Leppanen, M. Karttunen, R. A. Barrio, and K. Kaski, Phys. Rev. E 70, 066202 (2004); Braz. J. Phys. 34, 368 (2004).
[9] T. Callahan and E. Knobloch, Physica D 132, 339 (1999) and earlier references.

[10] R. FitzHugh, Biophys. J. 1, 445 (1961); J. Nagumo, S. Arimoto, and S. Yoshizawa, Proc. IRE 50, 2061 (1962).

[11] J. Rinzel and J. B. Keller, Biophys. J. 13, 1313 (1973).

[12] J. J. Tyson and J. P. Keener, Physica D 32, 327 (1988).

[13] A. Shinozaki and Y. Oono, Phys. Rev. E 48, 2622 (1993).

[14] We have checked that all of the results can be reproduced for smaller values of the parameters $D_{v}=5.0 \times 10^{-4}$ and $\gamma=2.5$.

[15] A. Aksimetiev, M. Fialkowski, and R. Holyst, Adv. Chem. Phys. 121, 141 (2002).

[16] T. Ohta, Prog. Theor. Phys. Suppl. 99, 425 (1989).

[17] T. Ohta and K. Kawasaki, Macromolecules 19, 2621 (1986).

[18] However, this is less accurate for an Fddd structure because the intensity of higher harmonics is not necessarily negligible as shown in Table II. 